Check for updates

Cite this: RSC Adv., 2018, 8, 28329

Received 1st July 2018

Accepted 23rd July 2018

DOI: $10.1039 / c 8 r a 05622 f$

rsc.li/rsc-advances

\section{Synthesis of a cationic polyacrylamide by a photocatalytic surface-initiated method and evaluation of its flocculation and dewatering performance: nano- $\mathrm{TiO}_{2}$ as a photo initiator}

\author{
Yongzhi Liu, ab Huaili Zheng, (D)*ab Yili Wang, ${ }^{c}$ Xinyu Zheng, ${ }^{\text {ab }}$ Moxi Wang, ${ }^{\text {ab }}$ Jie Ren ${ }^{\text {ab }}$
} and Chuanliang Zhao ${ }^{\mathrm{ab}}$

\begin{abstract}
In the face of complex water quality changes, the application of existing cationic polyacrylamide has been largely limited. In this study, a series of cationic polyacrylamides (TPADs) with excellent flocculation/ dewatering performance and low dosage were synthesized through photocatalytic surface initiation using acrylamide (AM) and acryloyloxyethyl trimethylammonium chloride (DAC) as monomers and nano$\mathrm{TiO}_{2}$ as an initiator. Characterization using Fourier transform infrared (FTIR) spectroscopy, nuclear magnetic resonance ( ${ }^{1} \mathrm{H}$ NMR) spectroscopy, thermogravimetric/differential scanning calorimetry (TG/ DSC) and scanning electron microscopy (SEM) was used to analyze the structural and morphological properties of TPADs. The initiation mechanism was described and the study on the properties of TPADs shows that the initiation method could obtain the copolymer with extra-high intrinsic viscosity. Furthermore, the flocculation and dewatering performance of TPADs and PADs were investigated in the micro-polluted low turbidity water flocculation test and sludge dewatering test. The application experimental results indicated that TPADs showed satisfactory turbidity removal and sludge dewatering performance by virtue of strong charge neutralization and a bridging effect. The excellent flocculation/ dewatering performance was attributed to the photocatalytic surface-initiated method and the nano$\mathrm{TiO}_{2}$ initiator. Therefore, it is expected to open up new initiation methods in the synthesis of polymeric flocculants for a broad variety of applications.
\end{abstract}

\section{Introduction}

Coagulation/flocculation is a very common and important purification technique to remove the suspended colloidal particles and various dissolved contaminants from water by charge neutralization, electrostatic patching, polymer bridging, and sweep flocculation. ${ }^{1}$ Coagulation/flocculation has been widely used in water treatment as well as sludge dewatering due to its high performance, cost-effectiveness and simple operation..$^{2-4}$ The critical factor that affects the performance of

${ }^{a}$ Key Laboratory of the Three Gorges Reservoir Region's Eco-Environment, Ministry of Education, Chongqing University, Campus B, 83 Shabei Street, Shapingba District, Chongqing 400045, PR China. E-mail: hlz6512@163.com; 1571709188@qq.com ${ }^{b}$ National Centre for International Research of Low-carbon and Green Buildings, Chongqing University, Chongqing 400045, PR China

${ }^{c}$ College of Environmental Science and Engineering, Research Center for Water Pollution Source Control and Eco-remediation, Beijing Forestry University, Beijing 100083, China

$\dagger$ Electronic supplementary information (ESI) available: Calculation method of $[\eta]$, ST, FCMC, SRF, and sludge floc properties in dewatering experiment. Details of used flocculant in flocculation and dewatering test. Floc photographs in flocculation experiment. See DOI: 10.1039/c8ra05622f coagulation/flocculation is the properties of the coagulants/ flocculants.

Coagulation is mainly induced by inorganic coagulants, such as polyaluminium chloride, polyferric sulfate, and polytitanium salt coagulants..$^{5-7}$ However, the use of these inorganic coagulants is limited due to the high dose requirement, low efficiency and large volume sludge production in practical applications. ${ }^{6,8}$ Therefore, polymeric flocculants including synthetic, natural and grafted flocculants with the properties of lower dose requirement, satisfactory solid-water separation performance and easy operation have been concerned and developed to improve the flocculation performance in water treatment. ${ }^{9-11}$ In order to obtain the polymeric flocculants with excellent properties, various methods for the synthesis of polymeric flocculants have been studied and applied recently.

Free radical copolymerization in aqueous solution has been widely used in the synthesis of flocculants due to its easy control and simple operation. ${ }^{12}$ Scholars have studied many initiation methods, including heat, ultrasound, plasma, microwave and ultraviolet (UV). Xu et al. reported that a new hydrophobically modified polymeric flocculant containing beta-cylodextrin was prepared by heat-initiated polymerization. ${ }^{13}$ Zhao et al. 
prepared a cationic flocculant with micro-block structure by ultrasound-initiated polymerization and evaluated its flocculation performance in the oily wastewater. ${ }^{\mathbf{1 4}}$ Sun et al. successfully synthesized an environment friendly chitosan-graft flocculant through plasma initiation and investigated the flocculation performance of grafted flocculant in low-algal-turbidity water. ${ }^{15}$

As far as we know, UV initiation is an eco-friendly and economic process, which can perform at the condition of lower reaction temperature, less initiator, and can achieve higher polymerization efficiency. ${ }^{\mathbf{1 6}}$ It has received extensive concern about the synthesis of polymeric flocculant and various materials. Li et al. prepared a cationic polyacrylamide by UV-initiated template copolymerization with superior sludge dewatering performance and a wide range of $\mathrm{pH}$ application. ${ }^{17}$ In recent years, photocatalytic surface-initiated method has been applied to synthesize inorganic/polymer hybrid nanocomposites. ${ }^{18}$ In initiated process, semiconductor nanoparticles are activated by the photon with high energy such as UV light and then generate electron holes or free radicals that can induce monomers polymerization near the surface of semiconductor nanoparticles. ${ }^{19,20}$ In addition, the photocatalytic surface-initiated method is more convenient and economical for the reason that it can realize polymerization without degassing but traditional UV initiation which requires the use of an excess pure $\mathrm{N}_{2}$ to drive off oxygen. ${ }^{21}$ Although photocatalytic surface-initiated polymerization has been studied in many reports, the method was mainly used for the synthesis of nanocomposites. ${ }^{22-24}$ Wang et al. reported that poly(methyl methacrylate) (PMMA) chains grafted $\mathrm{TiO}_{2}$ nanoparticles by photocatalytic polymerization had greatly different structure and properties comparing with traditional PMMA. ${ }^{25}$ In general, initiation method can improve the flocculation performance by modifying the surface structure of polymeric flocculants. ${ }^{17}$ Based on the above findings, the photocatalytic surface initiation is expected to apply for the synthesis of polymeric flocculants as a novel initiation technology.

To the best of our knowledge, no previous study has reported that photocatalytic surface initiation method was applied in the synthesis of cationic polymeric flocculant so far. In this work, nano-TiO ${ }_{2}$, one of the most promising semiconductor material with relatively high photocatalysis and stability, were used as initiator. ${ }^{26} \mathrm{AM}$ and DAC were used as monomers due to their high polymerization activity. ${ }^{27}$ Therefore, a series of cationic flocculants (TPADs) with distinctive cationic sequence structure, porous surface area and extra-high intrinsic viscosity by photocatalytic surface initiation using nano- $\mathrm{TiO}_{2}$ as initiator were prepared. FTIR, ${ }^{1} \mathrm{H}$ NMR, TG/DSC and SEM were used to characterize the polymer structure. Finally, flocculation ability was evaluated by jar test with micro-polluted lake water, and then sludge dewatering performance was studied.

\section{Experimental section}

\subsection{Materials and equipment}

Acrylamide (AM, 99 wt\% Lanjie Co., Ltd, Chongqing, China); acryloyloxyethyl trimethylammonium chloride (DAC, $75 \mathrm{wt} \%$, Guangchuangjing Import and Export Co., Ltd, shanghai,
China); nano titanium dioxide $\left(\mathrm{TiO}_{2}, 99.8 \%\right.$ metals basis, $25 \mathrm{~nm}$, anatase, hydrophilic, Aladdin chemical, Shanghai, China); photo-initiator 2,2'-azobis[2-(2-imidazolin-2-yl) propane]dihydrochloride (VA-044, Ruihong Biological Technology, Shanghai, China); Polyacrylamide (PAM, Lanjie Co., Ltd, Chongqing, China); Commercial cationic PAM (CPAM, $\mathrm{AM}$ and DAC copolymer, intrinsic viscosity $1450 \mathrm{mg} \mathrm{L}^{-1}$, Lanjie Co., Ltd, Chongqing, China).

Anhydrous ethanol and acetone were provided by Chuandong Chemical Company, Chongqing, China. All solutions in this study were prepared with ultrapure water (GWA-UN, General, Beijing, China). Fig. 1 shows a schematic of the experimental setup, which is composed of a water-cooled $500 \mathrm{~W}$ high-pressure $\mathrm{Hg}$ lamp assembly (main radiation wavelength, $365 \mathrm{~nm}$ ) and reaction vessels (Pyrex glass) coupled with rotating platforms. The high-pressure $\mathrm{Hg}$ lamp was powered by an adjustable power supply (Jiguang Special Lighting Electrical Factory, Shanghai, China). UV irradiation intensity was measured by an ultraviolet radiation meter (UV-A, HANDY, Beijing Normal University Photoelectric Instrument Factory, China).

\subsection{Synthesis of copolymers}

Cationic polymeric flocculants named as TPADs (TPAD1-6) were synthesized by photocatalytic surface-initiated polymerization using nano- $\mathrm{TiO}_{2}$ as initiator. The synthetic conditions are shown in Table 3. The desired amount of AM, DAC and a certain volume of ultrapure water were added to a $50 \mathrm{~mL}$ Pyrex glass vessel and then stirred until dissolving totally. Afterward, a predetermined amount of $\mathrm{TiO}_{2}$ was added. The nano-TiO particles were uniformly dispersed in mixed solution by using ultrasonic cleaner (KQ-500 DE, Kunshan ultrasonic instrument Co., Ltd, China). Then, the reaction vessel was sealed and exposed to UV irradiation in ambient temperature until the required reaction time. The irradiation intensity was kept at $1000 \mu \mathrm{m} \mathrm{cm}^{-2}$. During experiments, reaction vessels were set on the rotating platform with a rotation speed of $8 \mathrm{rpm}$ to make vessels receive UV-light regularly. After polymerization, the product was purified with excess acetone and then soaked in anhydrous ethanol for $24 \mathrm{~h}$. Finally, the product was dried in a vacuum oven at $50{ }^{\circ} \mathrm{C}$ until constant weight. PAD1-3

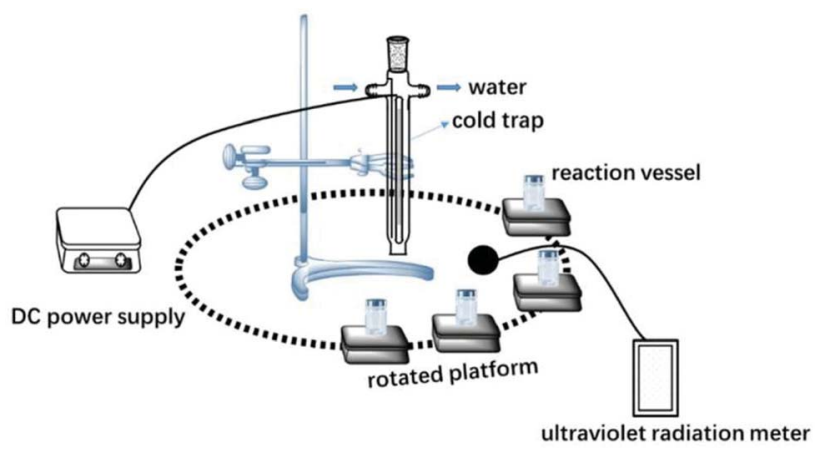

Fig. 1 Schematic of the experimental equipment. 
(copolymer of AM and DAC) were synthesized using VA-044 as photo initiator under degassing with pure $\mathrm{N}_{2}$ and VA-044 concentration was fixed at $0.5 \mathrm{wt} \%$.

\subsection{Characterization of copolymers}

In the section, PAM, PAD and TPADs after purification were ground into powder for characterization analysis. The FTIR spectra of the polymers were acquired using $\mathrm{KBr}$ pellets on a 550 Series II infrared spectrometer (Bruker Company, Switzerland) with wave numbers from 500 to $4000 \mathrm{~cm}^{-1}$. The ${ }^{1} \mathrm{H}$ NMR of the polymers were obtained by Advance-500 NMR spectrometer (Bruker Company, Germany) in deuterium oxide $\left(\mathrm{D}_{2} \mathrm{O}\right)$ as a solvent with tetramethylsilane as an internal standard. The thermal stability (TG/DSC) of the polymers were determined by a TG/DSC/1100LF instrument (Mettler, Switzerland) under argon atmosphere from $20{ }^{\circ} \mathrm{C}$ to $600{ }^{\circ} \mathrm{C}$ at a heating rate of $10{ }^{\circ} \mathrm{C} \mathrm{min}^{-1}$. The SEM images of the polymers were acquired by MIRA3 LMH (Tescan Trade, Shanghai) instrument to investigate their surface morphology. The BET surface areas were measured on ASAP $2020 \mathrm{M}+\mathrm{C}$ (Micromeritics, American).

\subsection{Flocculation test}

Raw water was taken from micro-polluted low-turbidity lake water in Chongqing University. The water quality parameters were listed in Table 1. Predetermined dose flocculants were added into $200 \mathrm{~mL}$ micro-polluted water. The $\mathrm{pH}$ of the solution was adjusted with both hydrochloric acid $\left(0.1 \mathrm{~mol} \mathrm{~L}^{-1}\right)$ and sodium hydroxide $\left(0.1 \mathrm{~mol} \mathrm{~L}^{-1}\right)$. The flocculation experiments were performed on a program-controlled jar test apparatus (TA6, Hengling Technology Co., Ltd, China). The flocculation procedure includes three steps. Firstly, rapidly stirred $200 \mathrm{rpm}$ for $2 \mathrm{~min}$ to fully collide between flocculants with suspension particles; secondly, slowly stirred at $60 \mathrm{rpm}$ for $10 \mathrm{~min}$ to make the flocs sufficiently growth; finally, the formed flocs free settled for $5 \mathrm{~min}$. To evaluate the flocculation performance, supernatant turbidity and $\mathrm{UV}_{254}$ were measured. The supernatant turbidity at depth $2 \mathrm{~cm}$ below the water surface was measured with a HACH (HACH 2100Q, American Hach Company) turbidity meter. The absorbance was measured with a double beam UV-vis spectrophotometer (T6 New Century, Beijing General Instrument Co., Ltd, China) at $\lambda$ $=254 \mathrm{~nm}$. $\mathrm{UV}_{254}$ was used to represent the concentration of natural organic matter (NOM) in the micro-polluted water. ${ }^{28}$

The turbidity removal efficiency (TRE) was calculated by following formula (1).

$$
\mathrm{TRE}=\frac{N_{0}-N_{1}}{N_{0}}
$$

Table 1 Characteristics of the micro-polluted lake water sample

\begin{tabular}{lllll}
\hline $\begin{array}{l}\text { Raw water quality } \\
\text { parameters }\end{array}$ & $\begin{array}{l}\mathrm{COD} \\
\left(\mathrm{mg} \mathrm{L}^{-1}\right)\end{array}$ & $\begin{array}{l}\text { Turbidity } \\
(\mathrm{NTU})\end{array}$ & $\begin{array}{l}\mathrm{UV}_{254} \\
\text { absorbance }\end{array}$ & $\mathrm{pH}$ \\
\hline Value & $20-30$ & $17-21$ & $0.115-0.116$ & $7.5-8.0$
\end{tabular}

where $N_{0}$ is the initial turbidity, $N_{1}$ is the turbidity after treatment.

\subsection{Dewatering test}

The sludge was sampled from the secondary sedimentation tank in Jingkou Wastewater Treatment Plant in Chongqing, China. The sludge properties were given in Table 2. A certain amount of flocculant was added into $100 \mathrm{~mL}$ sludge. The $\mathrm{pH}$ of the sludge was adjusted with both hydrochloric acid $\left(0.1 \mathrm{~mol} \mathrm{~L}^{-1}\right)$ and sodium hydroxide $\left(0.1 \mathrm{~mol} \mathrm{~L}^{-1}\right)$. The dewatering tests were carried out on a program-controlled jar test apparatus (TA6, Hengling Technology Co., Ltd, China). The mixing procedure includes three steps: rapid mix period for $30 \mathrm{~s}$ at $200 \mathrm{rpm}$ followed by slow stirring at $50 \mathrm{rpm}$ for $2 \mathrm{~min}$, and then the formed flocs free settled for $5 \mathrm{~min}$. The dewatering ability of the synthesized copolymer was evaluated by measuring the properties of sludge including supernatant turbidity (ST), filter cake moisture content (FCMC), specific resistance to filtration (SRF) and sludge floc size.

\section{Results and discussion}

\subsection{Property comparison for PADs and TPADs}

The polymerization conditions and the intrinsic viscosity $([\eta])$ of the copolymers are listed in Table 3 . As shown in Table 3, when DAC feed ratio was $30 \%,[\eta]$ of TPADs (TPAD1-TPAD4) showed an increasing trend with the increase in the amount of $\mathrm{TiO}_{2}$, reaching the maximum value at $\mathrm{TiO}_{2}$ content $(0.250 \mathrm{wt} \%$ o $)$ and then slightly decreased. Correspondingly, $M_{\mathrm{r}}$ of TPADs exhibited a similar trend. This phenomenon was because excess $\mathrm{TiO}_{2}$ would rapidly generate superfluous the valence-band hole $\left(\mathrm{h}^{+}\right)$ and $\mathrm{OH}$ - to induce the intermolecular crosslink reaction, causing poor dissolution properties and thus reducing the intrinsic viscosity. ${ }^{29,30}$ Moreover, the reaction time forming gel product of $\mathrm{TiO}_{2}$ content $0.375 \mathrm{wt} \%$ and $0.500 \mathrm{wt} \%$ was shorter than that of $\mathrm{TiO}_{2}$ content $0.125 \mathrm{wt} \%$ and $0.25 \mathrm{wt} \%$. This result was attributed to that the increase of $\mathrm{TiO}_{2}$ content resulted in the faster polymerization rate and a shorter polymerization time. ${ }^{19}$ However, when the $\mathrm{TiO}_{2}$ content was fixed at $0.25 \mathrm{wt} \%$, $[\eta]$ of TPADs (TPAD5, TPAD1 and TPAD6) gradually decreased with the increase of DAC feed ratio. AM is the most reactive monomers to undergo radical polymerization and thus building up high intrinsic viscosity. ${ }^{9}$ Because the total monomer concentration was fixed at $25 \mathrm{wt} \%$ in the experiment, with the increase of DAC feed ratio, the AM feed ratio correspondingly decreased then the activity of the system declined. Moreover, $[\eta]$ of TPADs was higher than PADs under same feed ratio, which was probably because $\mathrm{TiO}_{2}$ exhibited high quantum yield in the photocatalytic surface initiation method.

\subsection{Characterization}

3.2.1 FTIR spectra. The FTIR spectra of PAM, PAD1, and TPAD2 are shown in Fig. 2. In the FTIR spectra of TPAD2, the adsorption peaks at 3454 and $2952 \mathrm{~cm}^{-1}$ were attributed to the stretching vibration of $-\mathrm{NH}_{2}$ groups of amide in $\mathrm{AM}$ unit and the asymmetric stretching vibration of $-\mathrm{CH}_{2}$ - in AM and DAC 
Table 2 Characteristics of the Sludge Sample

\begin{tabular}{llllll}
\hline Indicator & Moisture content $(\%)$ & Mass density $\left(\mathrm{kg} \mathrm{L}^{-1}\right)$ & SRF $\left(10^{12} \mathrm{~cm} \mathrm{~g}^{-1}\right)$ & Zeta potential $(\mathrm{mV})$ & $\mathrm{pH}$ \\
\hline Value & 97.31 & 1.020 & 1.3852 & -14.06
\end{tabular}

Table 3 Polymerization conditions and intrinsic viscosity of the copolymers

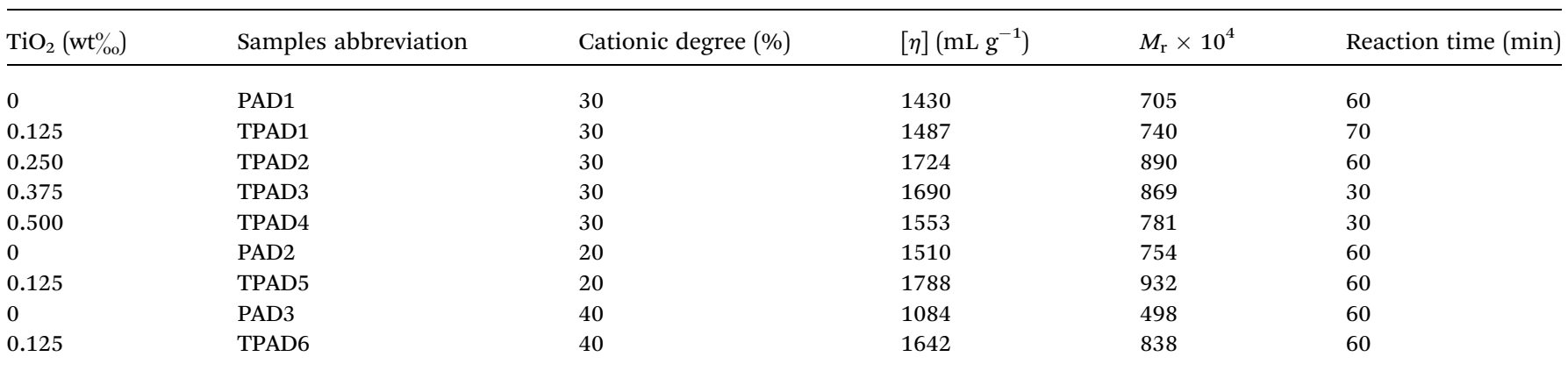

monomer. ${ }^{31}$ The adsorption peaks at 1659 and $1454 \mathrm{~cm}^{-1}$ were for $\mathrm{C}=\mathrm{O}$ stretching vibration and $-\mathrm{CH}_{2}-$ flexural vibrations, respectively. The characteristic adsorption peaks at 1737,1171 and $953 \mathrm{~cm}^{-1}$ were assigned to the $\mathrm{C}=\mathrm{O}, \mathrm{C}-\mathrm{O}$ asymmetric stretching vibration and $\mathrm{N}^{+}\left(\mathrm{CH}_{3}\right)_{3}$ stretching vibration in DAC units, respectively. ${ }^{29,30}$ Compared with the spectra of PAM, PAD1 and TPAD2, the corresponding peaks of main groups in AM and DAC were all observed in the spectra of PAD1 and TPAD2. The comparative analysis indicated that the copolymer of AM and DAC for PAD1 and TPAD2 were successfully synthesized. Although the adsorption peaks of PAD1 and TPAD2 were almost identical, the intensity for the $\mathrm{N}^{+}\left(\mathrm{CH}_{3}\right)_{3}$ characteristic adsorption peak in $953 \mathrm{~cm}^{-1}$ was different under the same cationic degree. The intensity in $953 \mathrm{~cm}^{-1}$ of TPAD2 were slightly stronger than that of PAD1. According to our previous works, template assembly method could cause the change of the $\mathrm{N}^{+}$ $\left(\mathrm{CH}_{3}\right)_{3}$ characteristic adsorption peak in FTIR spectrum. ${ }^{31,32}$

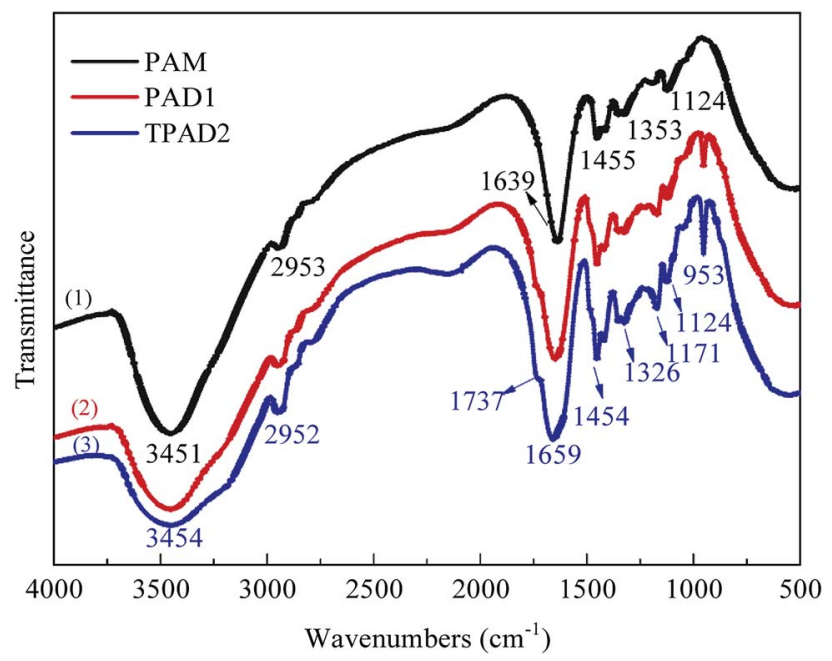

Fig. 2 FTIR spectra of (1) PAM, (2) PAD1, and (3) TPAD2.
Similarly, the above phenomenon may be related to the different cationic sequence distribution in TPAD2 that was synthesized by the photocatalytic surface-initiated polymerization. ${ }^{32}$ The assumption will be further explored in the following parts.

3.2.2 ${ }^{1} \mathrm{H}$ NMR spectra. The ${ }^{1} \mathrm{H}$ NMR spectra analysis was performed to further study the copolymer structure. Fig. 3 depicts the ${ }^{1} \mathrm{H}$ NMR spectra of PAM, PAD1 and TPAD2. In the ${ }^{1} \mathrm{H}$ NMR spectrum of TPAD2, the asymmetric peaks at $\delta=1.61$ and $\delta=2.18 \mathrm{ppm}$ were attributed to the protons of $-\mathrm{CH}_{2}\left(\mathrm{a}_{1}\right)$ and $-\mathrm{CH}\left(\mathrm{b}_{1}\right)$ groups. The characteristic peaks at $\delta=4.53, \delta=3.71$ and $\delta=3.18 \mathrm{ppm}$ were attributed to protons of $-\mathrm{O}-\mathrm{CH}_{2}$ (c), $-\mathrm{CH}_{2}-\mathrm{N}^{+}-(\mathrm{d})$, and $-\mathrm{N}^{+}-\left(\mathrm{CH}_{3}\right)_{3}(\mathrm{e})$, respectively. ${ }^{29}$ The appeared adsorption peaks at $\delta=4.01$ (f) and $\delta=3.47(\mathrm{~g}) \mathrm{ppm}$ were attributed to the $\mathrm{H}_{\mathrm{c}}$ and $\mathrm{H}_{\mathrm{d}}$ protons of copolymer. ${ }^{16}$ In addition, the spectra of PAD1 and TPAD2 showed slight chemical shift of at $\mathrm{H}_{\mathrm{a} 1}$ and $\mathrm{H}_{\mathrm{b} 1}$ protons compared with the spectrum of PAM at $\mathrm{H}_{\mathrm{a}}$ and $\mathrm{H}_{\mathrm{b}}$ protons. It was explained that the copolymerization

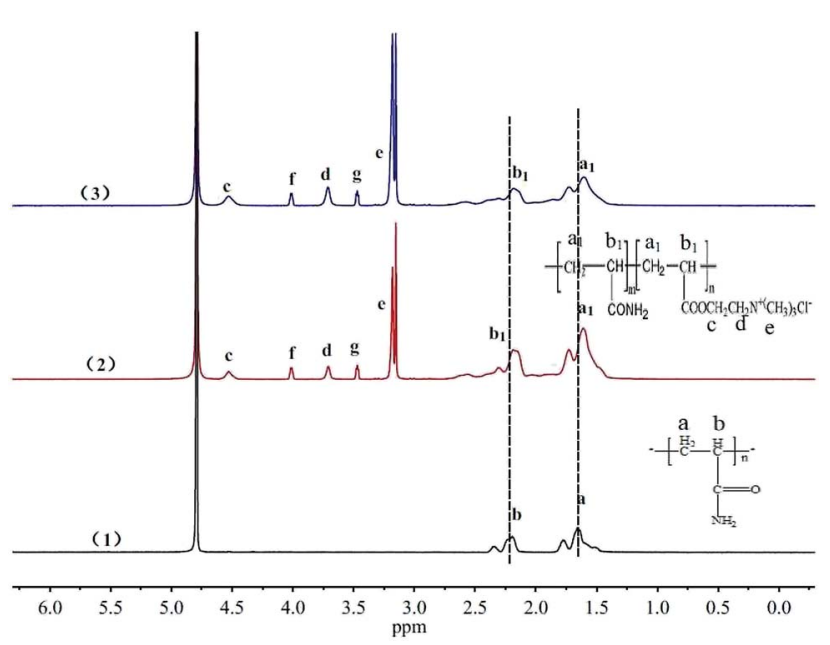

Fig. $3{ }^{1} \mathrm{H}$ NMR spectra of (1) PAM, (2) PAD1, and (3) TPAD2. 

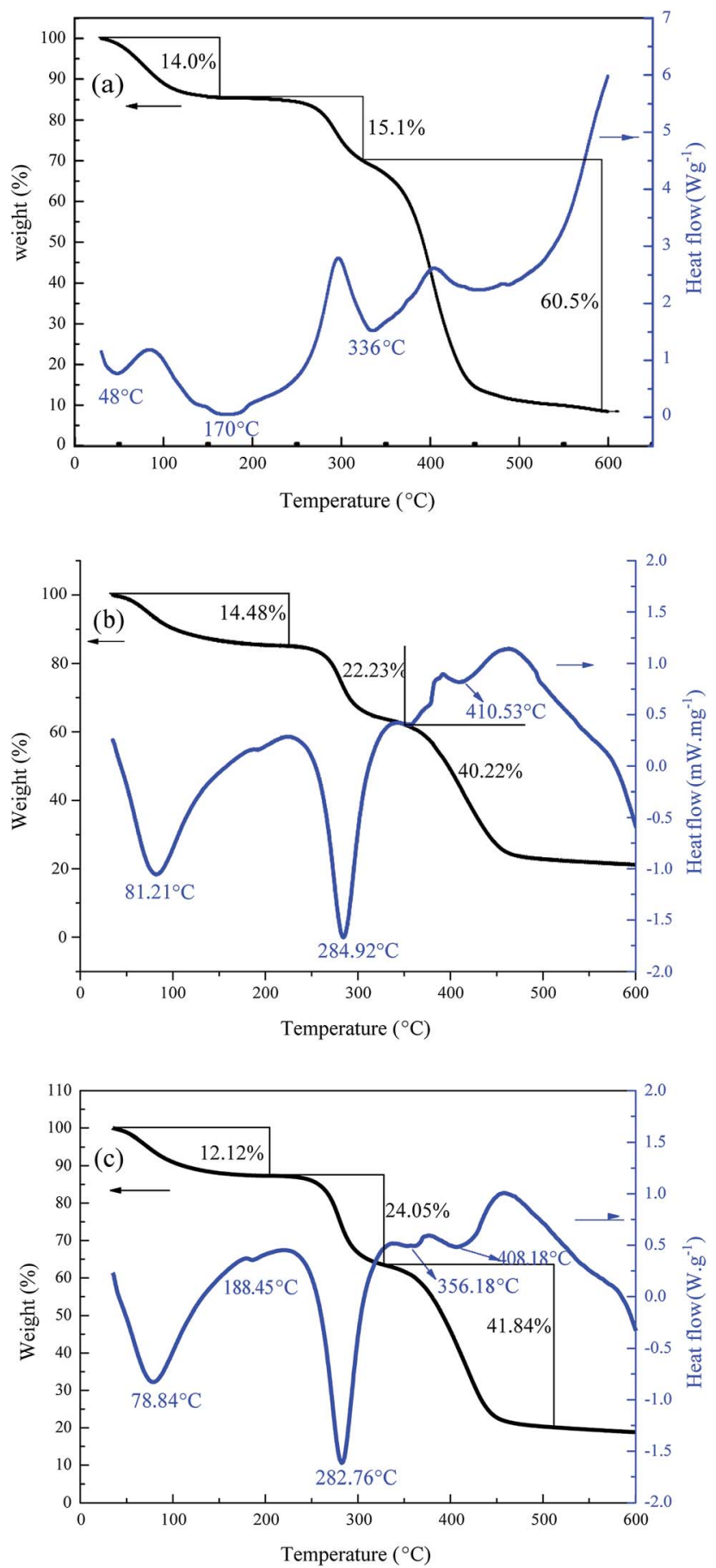

Fig. 4 TG/DSC analysis of (a) PAM, (b) PAD1, and (c) TPAD2.

of AM and DAC caused the chemical environmental change of the $\mathrm{H}_{\mathrm{a} 1}$ and $\mathrm{H}_{\mathrm{b} 1}$ protons. $^{2}$ These aforementioned new peaks and peak shift suggested that PAD1 and TPAD2 were successfully synthesized. Furthermore, in order to investigate the minor difference between PAD1 and TPAD2, the peak area of $\mathrm{H}_{\mathrm{a} 1}, \mathrm{H}_{\mathrm{b} 1}$, $\mathrm{H}_{\mathrm{d}+\mathrm{g}}$ in the spectra of PAD1 and TPAD2 was integrated. The peak area ratios of $\mathrm{H}_{\mathrm{a} 1}, \mathrm{H}_{\mathrm{b} 1}, \mathrm{H}_{\mathrm{d}+\mathrm{g}}$ in PAD1 and TPAD2 were $1: 0.469: 0.193$ and $1: 0.443: 0.212$. It was found that the ratio of $\mathrm{H}_{\mathrm{d}+\mathrm{g}}$ in TPAD2 was bigger than that in PAD1. The phenomenon was also because the TPAD2 had a different cationic sequence distribution structure compared with PAD1 and hence the adsorption intensity $\left(\mathrm{H}_{\mathrm{d}+\mathrm{g}}\right)$ of cationic monomer DAC was improved. ${ }^{17}$

3.2.3 TG/DSC characterization. Fig. 4 shows the thermal stability of PAM, PAD1, and TPAD2. As shown in Fig. 4, the three polymers had three main weight loss stages. The first stage (32.67-163.33 ${ }^{\circ} \mathrm{C}, 14.0 \%$ for PAM; $39.25-208.81{ }^{\circ} \mathrm{C}, 14.48 \%$ for PAD1; $37.01-205.86{ }^{\circ} \mathrm{C}, 12.12 \%$ for TPAD2) was due to the loss of absorbed water. The second stage $\left(163.33-323.67{ }^{\circ} \mathrm{C}, 15.1 \%\right.$ for PAM; $208.81-345.60{ }^{\circ} \mathrm{C}, 22.23 \%$ for PAD1; $205.86-327.13^{\circ} \mathrm{C}$, $24.05 \%$ for TPAD2) was attributed to the imidization of amide groups and the thermal decomposition of methyl from quaternary ammonium. ${ }^{15}$ The final stage $\left(323.67-592.67{ }^{\circ} \mathrm{C}\right.$, $60.5 \%$ for PAM; $345.60-534.50{ }^{\circ} \mathrm{C}, 40.22 \%$ for PAD1; $327.13-$ $511.737^{\circ} \mathrm{C}, 41.84 \%$ for TPAD2) was the thermal decomposition of the polymer backbone. ${ }^{33}$ From DSC curve in Fig. 4(c), there was two sight endothermic peaks at 356.18 and $408.18{ }^{\circ} \mathrm{C}$. However, there was only one endothermic peak at $410.53{ }^{\circ} \mathrm{C}$ in Fig. 4(b). The results indicated that TPAD2 had a two-phase structure. Based on previous work, it has reported that the change of copolymer sequence distribution could cause the change of thermal stability. ${ }^{34}$ This could well interpret the above phenomenon. In conclusion, TG/DSC analysis indicated that PAD1 and TPAD2 had a good thermal stability and further demonstrated TPAD2 contained a distinctive sequence structure.

3.2.4 SEM analysis and physical properties of the copolymers. SEM images and calculated fractal dimension are illustrated in Fig. 5, which directly shows the surface morphology of PAD1, TPAD2 and TPAD4. Obviously, TPAD2 (Fig. 5(b)) and TPAD4 (Fig. 5(c)) had plenty of holes, uneven surface structure and huge surface area. But, PAD1 had a regular structure with smooth surface morphology. Previous study involving UV light can modify the polymer surface to increase the surface porosity and surface area ${ }^{35}$ In this study, PAD1 was also synthesized by UV initiation but the porous surface was not observed as shown in Fig. 5(a). This indicated that the porous structure of TPADs may attribute to nano- $\mathrm{TiO}_{2}$. In the polymerization reaction, nano- $\mathrm{TiO}_{2}$ particles were evenly distributed in mixed solution and then occupied a certain spatial position. After purification using excess acetone, most of $\mathrm{TiO}_{2}$ particles were removed resulting in forming of the porous structure in TPADs surface. Therefore, nano- $-\mathrm{TiO}_{2}$ was not only the initiator of polymerization but also the sacrificing template. ${ }^{36,37}$ Moreover, Image-Pro Plus 6.0 software was used to calculate the fractal dimension which could be a proof for further investigating the surface morphology. The fractal dimensions of PAD1, TPAD2 and TPAD4 were 1.366, 1.937 and 2.208, respectively. The larger fractal dimension of TPAD2 and TPAD4 than PAD1 showed TPADs had a porous surface structure. In addition, the physical parameters of the microspheres were presented in Table S1. $\dagger$ Compared with PAD1, TPAD2 and TPAD4 had a larger specific surface that can improve the flocculation and dewatering performance of TPADs. ${ }^{38}$ It also indirectly demonstrated the role of nano- $\mathrm{TiO}_{2}$ photo catalysis on structural properties of copolymers. 

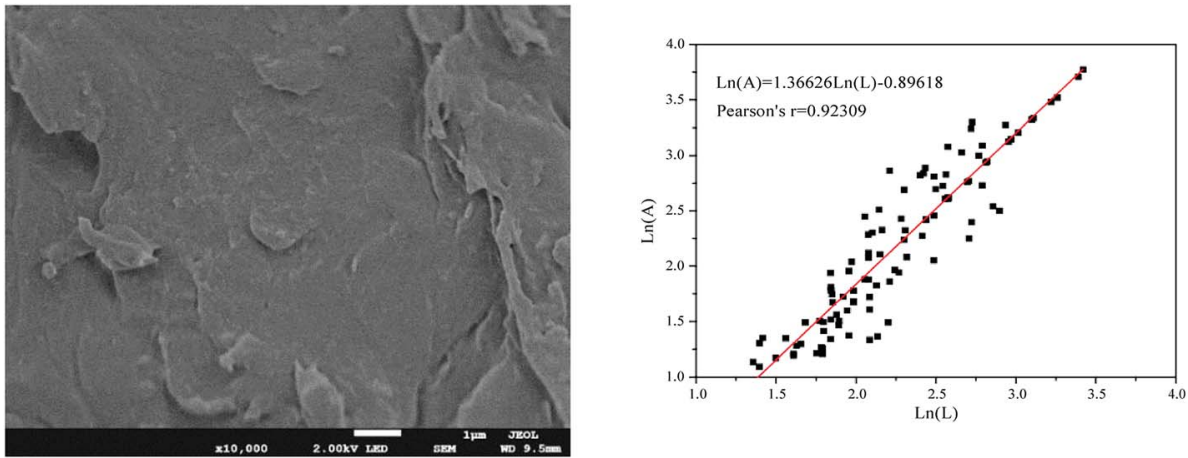

(a)
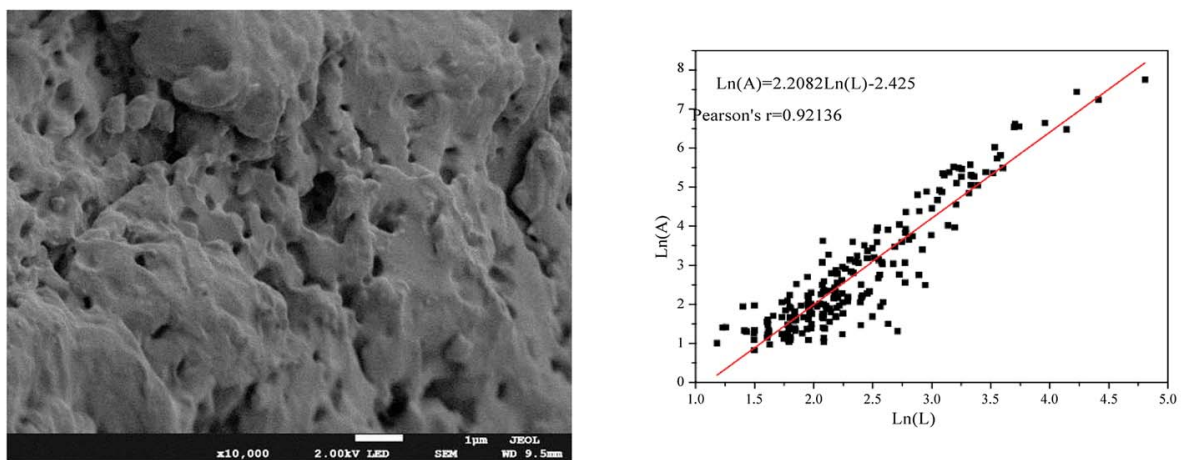

(b)
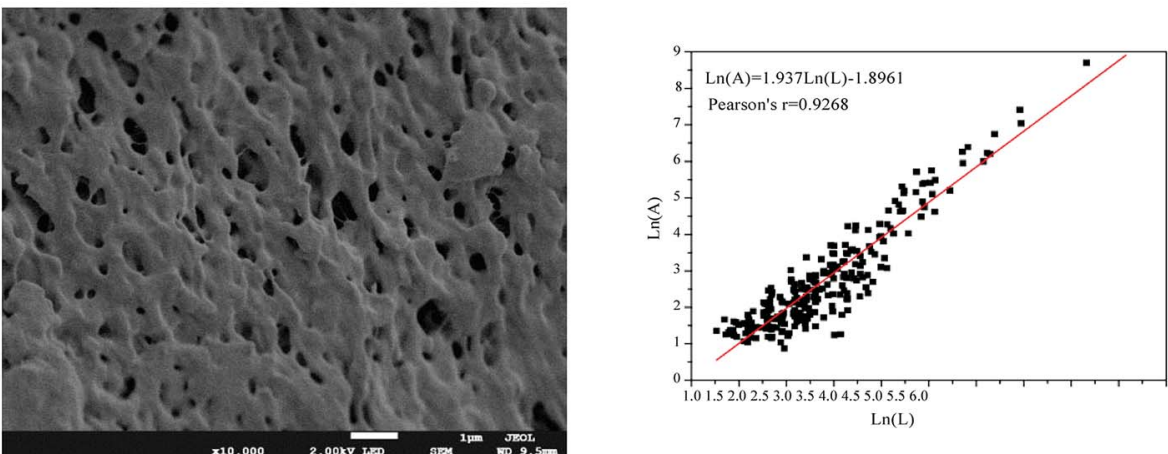

(c)

Fig. 5 SEM images of (a) PAD1, (b) TPAD2, and (c) TPAD4.

$$
\begin{aligned}
& \mathrm{TiO}_{2} \longrightarrow \mathrm{hv} \longrightarrow \mathrm{TiO}_{2}\left(\mathrm{~h}^{+}\right)+\mathrm{TiO}_{2}\left(\mathrm{e}^{-}\right) \\
& \mathrm{TiO}_{2}\left(\mathrm{e}^{\mathrm{e}}\right)+\mathrm{O}_{2}+\mathrm{H}_{2} \mathrm{O}_{2} \longrightarrow \mathrm{OH}
\end{aligned}
$$$$
\mathrm{TiO}_{2}\left(\mathrm{~h}^{+}\right)+\mathrm{H}_{2} \mathrm{C}=\mathrm{CH}+\mathrm{H}_{2} \mathrm{C}=\mathrm{CH} \text { chain propagation }-(\mathrm{H}-\mathrm{C}-\mathrm{CH})\left(\mathrm{H}-\mathrm{C}-\mathrm{CH}-\mathrm{H}_{2} \mathrm{C}-\mathrm{CH}\right.
$$$$
\begin{array}{llll}
\mathrm{C}=\mathrm{O} & \mathrm{COO}\left(\mathrm{CH}_{2}\right)_{2} \mathrm{~N}^{+}\left(\mathrm{CH}_{3}\right)_{3} \mathrm{Cl} & \stackrel{\mathrm{C}=\mathrm{O}}{\mathrm{C}} & \mathrm{COO}\left(\mathrm{CH}_{2}\right)_{2} \mathrm{~N}^{+}\left(\mathrm{CH}_{3}\right)_{3} \mathrm{Cl} \\
\mathrm{NH}_{2} & & \mathrm{NH}_{2} &
\end{array}
$$

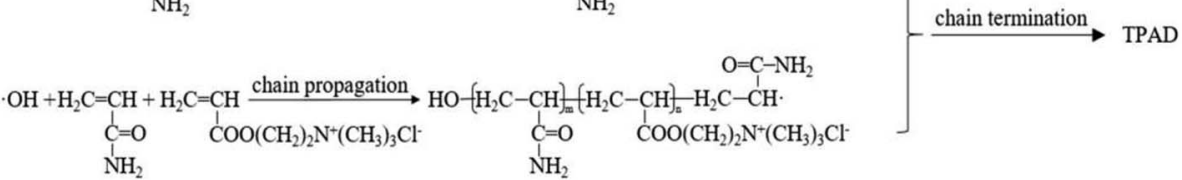

Fig. 6 The copolymerization mechanism schematic. 


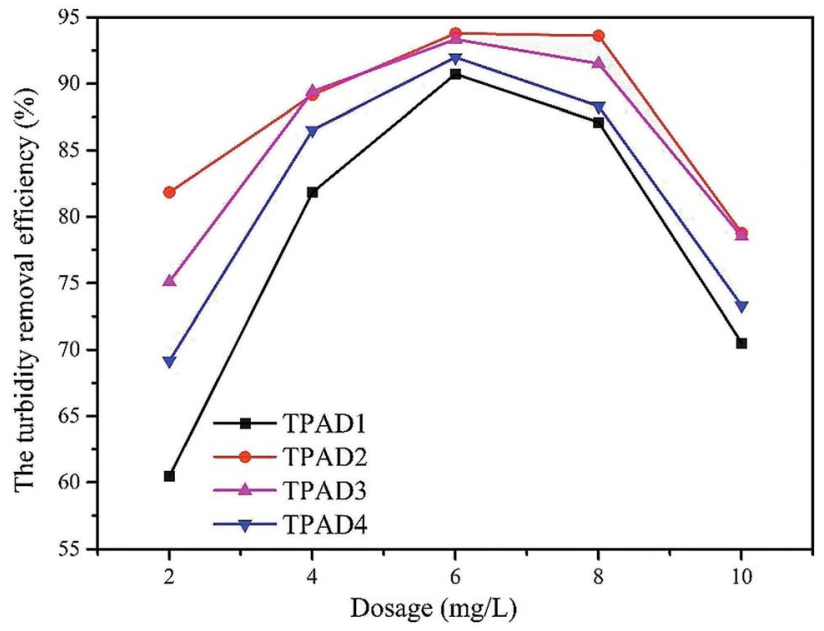

Fig. 7 Effect of flocculant category on the turbidity removal efficiency as a function of flocculant dosage.

\subsection{Initiation mechanism in the copolymerization}

As the initiation mechanism depicted in Fig. 6, the surface of nano- $\mathrm{TiO}_{2}$ particles were excited by the $365 \mathrm{~nm}$ UV light to produce the conduction-band electron $\left(\mathrm{e}^{-}\right)$and the valenceband hole $\left(\mathrm{h}^{+}\right){ }^{18}$ The valence-band hole could abstract a hydrogen atom from monomer AM and DAC generating macroradical (AM - or DAC · ) that reacted with other monomer to proceed chain propagation. ${ }^{39}$ In addition, according to Ni's report $^{19}$ the system would produce hydroxyl radicals $(\mathrm{OH} \cdot)$ that could also initiate monomer AM and DAC copolymerization by opening double band according with classic radical polymerization mechanisms. ${ }^{40}$ Hence, the polymerization system existed two kinds of initiation mechanism as shown in Fig. 6.

\subsection{Flocculation test}

The above parts have mentioned that TPADs had a different cationic sequence distribution structure, extra-high intrinsic viscosity and prodigious surface area compared with PADs. In order to further investigate the flocculation performance, TPAD1-TPAD4, PAD1 (almost identical intrinsic viscosity with TPAD1) and CPAM (commercial cationic polyacrylamide) were selected for treating the micro-polluted lake water. The properties of these flocculants were listed in Table S1. $\dagger$ The turbidity removal efficiency (TRE), $\mathrm{UV}_{254}$ and zeta potential were regarded as main indexes to investigate the influence of the dosage and $\mathrm{pH}$ for various flocculants.

3.4.1 The effect of flocculants category. Fig. 7 shows that the effect of dosage on TRE for TPAD1-TPAD4. In Fig. 7, the TRE of TPAD1-TPAD4 initially increased as increased dosage and then decreased when flocculants dosage was above $6 \mathrm{mg} \mathrm{L}^{-1}$. The maximum of TRE for TPAD1-TPAD4 was 90.72\%, 93.79\%, 93.34\%, and $91.98 \%$ at optimum dosage $6 \mathrm{mg} \mathrm{L}^{-1}$, respectively. The optimum flocculation performance for the four flocculants was sorted as following: TPAD2 > TPAD3 > TPAD4 > TPAD1. The trend was proportional to $[\eta]$ of copolymer. In this study, same DAC feed ratio of the four flocculants narrowed their gap about
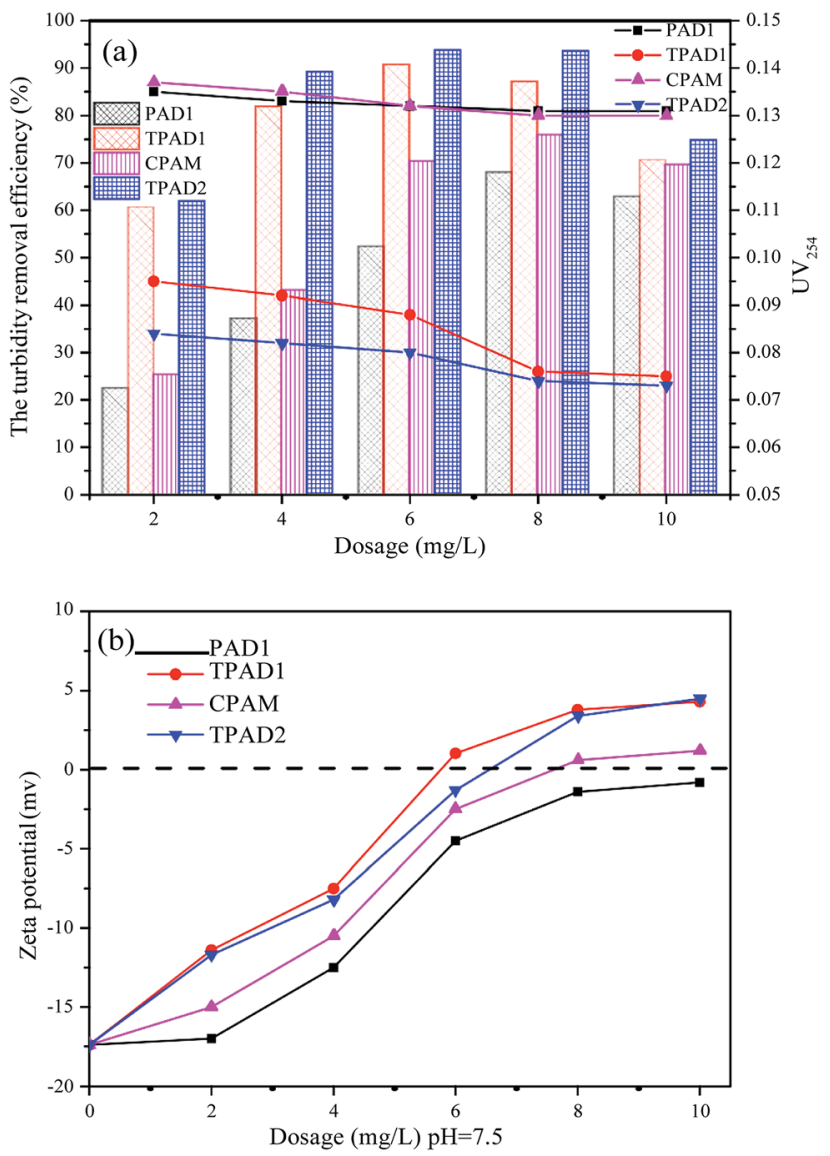

Fig. 8 The effect of dosage on (a) the turbidity removal efficiency and $\mathrm{UV}_{254}$ and (b) zeta potential.

the charge neutralization effect on flocculation mechanism. Hence, $[\eta]$ became a critical factor affecting the flocculation performance. Polymeric flocculants with relatively high $[\eta]$ could expand and extend more chain conformation in water reducing the gap between flocculants and colloid particles. Therefore, flocculants with relatively higher $[\eta]$ could be more likely to collide with colloid particles, and then enhanced the bridging effect to obtain better flocculation efficiency. ${ }^{\mathbf{4 1 , 4 2}}$

3.4.2 The effect of dosage. Fig. 8(a) shows the effect of flocculants dosage on the TRE and $\mathrm{UV}_{254}$ for PAD1, TPAD1, CPAM and TPAD2. As shown in the histogram of Fig. 8(a), the TRE for the four flocculants initially increased with the increase of dosage, and then gradually decreased with further increase in dose after reached certain dosage $\left(67.94 \%\right.$ at dosage $8 \mathrm{mg} \mathrm{L}^{-1}$, $75.87 \%$ at dosage $8 \mathrm{mg} \mathrm{L}{ }^{-1}, 90.72 \%$ at dosage $6 \mathrm{mg} \mathrm{L}^{-1}, 93.79 \%$ at dosage $6 \mathrm{mg} \mathrm{L}^{-1}$ ). This was consistent with the previous works by Liao and Yang ${ }^{4,43}$ who reported that flocculants were insufficient to occur charge neutralization and bridging effect effectively when flocculants dosage was low. However, excess dosage would cause the destabilization of flocs and cage effect, which restrained the growth of flocs then reduced flocculation efficiency. In addition, the optimum TRE for TPAD1 and TPAD2 was higher than CPAM and PAD1. Meanwhile, TPAD1 and TPAD2 had a lower optimum dosage than PAD1 and CPAM. 
The effect of flocculants dosage on $\mathrm{UV}_{254}$ is shown in the line chart of Fig. 8(a). For CPAM and PAD1, the $\mathrm{UV}_{254}$ was almost unchanged at about 0.13 , but the $\mathrm{UV}_{254}$ gradually decreased with the increase of TPAD1 and TPAD2 dosage and then approached a plateau at about 0.06. And the formed floc after TPAD2 flocculation was large and compact, whereas the formed floc of CPAM was tiny and loose as shown in Fig. S1. $\dagger$ These results indicated that the properties of TPADs including the different cationic sequence distribution structure, extra-high intrinsic viscosity and great surface area were conducive to improve flocculation performance. ${ }^{17}$ On one hand, the porous structure and huge surface area of TPADs were good for enhancing the bridging effect by increasing the probability of collision between flocculants and colloidal particles. ${ }^{27}$ On the other hand, the relatively high $[\eta]$ for TPAD1 and TPAD2 could also enhance the bridging effect then could improve flocculation efficiency.

In order to further explain the above mentioned phenomenon, the supernatant zeta potentials were measured. As shown in Fig. 8(b), with the increase of dosage, the zeta potentials gradually increased from negative to positive for the three flocculants except for PAD1 $\left(-0.8 \mathrm{mV}\right.$ at $\left.10 \mathrm{mg} \mathrm{L}^{-1}\right)$. In addition, the zeta potentials for TPAD1 and TPAD2 were higher than that
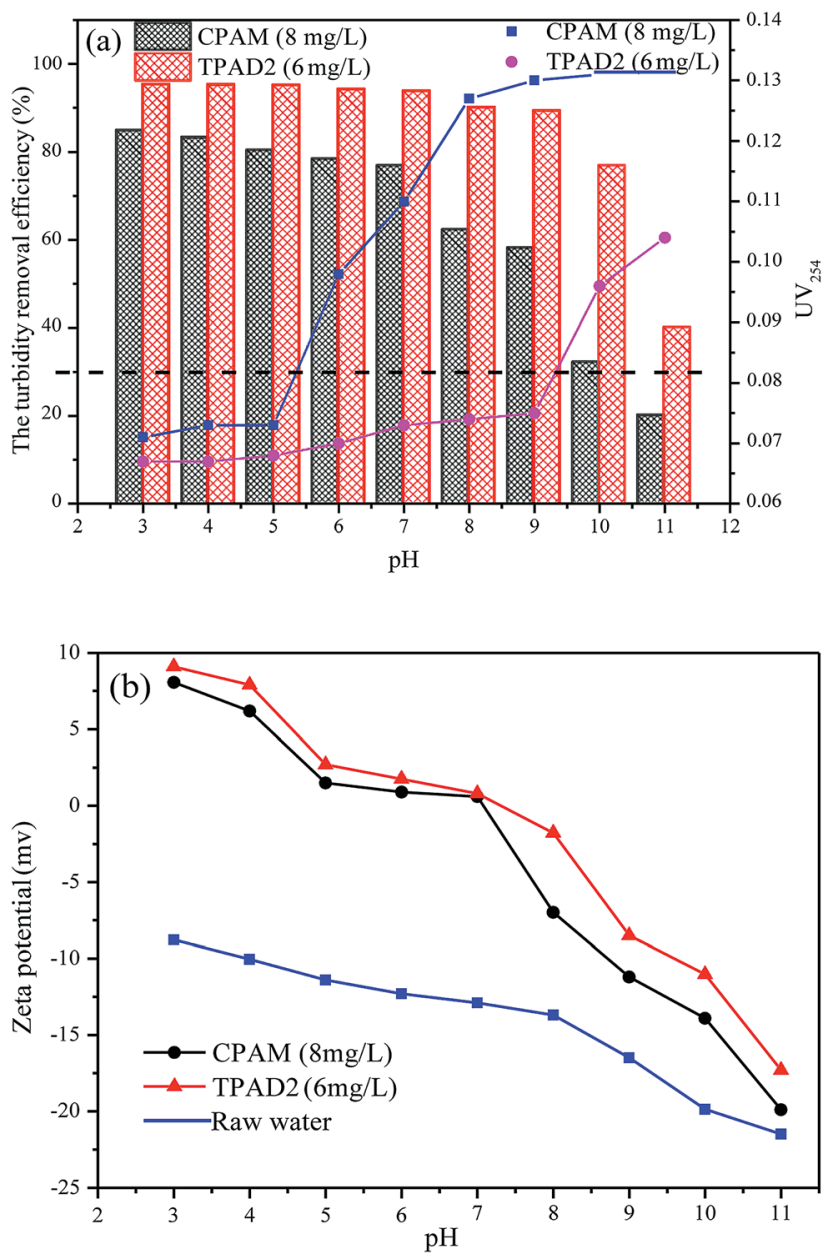

Fig. 9 The effect of $\mathrm{pH}$ on (a) the turbidity removal efficiency and $\mathrm{UV}_{254}$, and (b) zeta potential. for PAD1 and CPAM at the same dosage. The results demonstrated that the charge neutralization effect played a critical role in flocculation and TPAD1-2 had stronger charge neutralization ability and then could obtain better flocculation performance than PAD1 and CPAM. ${ }^{44}$ Moreover, the zeta potential for PAD1, CPAM, TPAD1 and TPAD2 at optimum dosage was -1.4, 0.6, $1.03,-1.3 \mathrm{mV}$, respectively. The negative zeta potential for PAD1 and TPAD2 at optimum dosage indicated that the bridging effect had a non-ignorable effect on flocculation in addition to the charge neutralization. ${ }^{45}$ In conclusion, the flocculation experiment revealed that the synthesized flocculants (TPAD1 and TPAD2) by the photocatalytic surface initiation polymerization had higher charge neutralization ability and were superior to the flocculants (PAD1 and CPAM) in the removal of turbidity and $\mathrm{UV}_{254}$.

3.4.3 The effect of $\mathbf{p H}$. In the flocculation process, $\mathrm{pH}$ was also a very important parameter to affect flocculation performance. Fig. 9 shows the flocculation ability and the change of supernatant zeta potential in the $\mathrm{pH}$ range of 3.0-11.0. As shown in Fig. 9(a), with the increased $\mathrm{pH}$ from 3.0 to 9.0, the TRE and $\mathrm{UV}_{254}$ after adding TPAD2 had slight changes (from $95.2 \%$ to $89.28 \%$; from 0.067 to 0.075 ). However, CPAM showed poor flocculation performance ( $\mathrm{TRE}<76 \%, \mathrm{UV}_{254}>0.11$ ) when $\mathrm{pH}$ value was greater than 7.0. This indicated that TPAD2 was more independent on the $\mathrm{pH}$ than CPAM. In addition, the flocculation performance of the two flocculants gradually decreased with the increase of $\mathrm{pH}$ in an alkaline environment.

As shown in Fig. 9(b), the zeta potential of the raw water continually decreased from -8.78 to $-21.5 \mathrm{mV}$ with the increased $\mathrm{pH}$ value from 3.0 to 11.0 . When the $\mathrm{pH}$ value below 5.0 or above 8.0, the zeta potential after flocculation with TPAD2 and CPAM presented excess positive or negative charge. This indicated that charge neutralization was not main flocculation mechanism under strong acid or strong alkaline environment due to excess positive charge or negative charge of raw water causing strong electrostatic repulsion between flocculants and colloidal particles. ${ }^{46}$ Therefore, TPAD2 with higher $[\eta]$ had better flocculation performance by the adsorption bridge and sweep flocculation than CPAM at pH below 5.0 or above 8.0. Moreover, the surface porosity of TPAD2 was also beneficial for the bridging effect by increasing the probability of collision between flocculants and colloidal particles. When $\mathrm{pH}$ increased from 5.0 to 8.0, the zeta potential after flocculation with TPAD2 was slightly higher than CPAM. The result demonstrated that TPAD2 was more efficient for the charge neutralization effect than CPAM and thus flocculation performance of TPAD2 was superior to CPAM at $\mathrm{pH}=5.0-8.0 . .^{34,47}$

\subsection{Dewatering test}

Considering that cationic polymeric flocculant is widely applicable on sludge dewatering. ${ }^{48}$ In this study, the dewatering abilities of PAD1 and TPAD1 (similar intrinsic viscosity for PAD1 and TPAD1) were compared taking ST, FCMC, SRF, zeta potential and flocs size as the main evaluation index. The effects of dosage and initial sludge $\mathrm{pH}$ were studied on the dewatering experiment. 

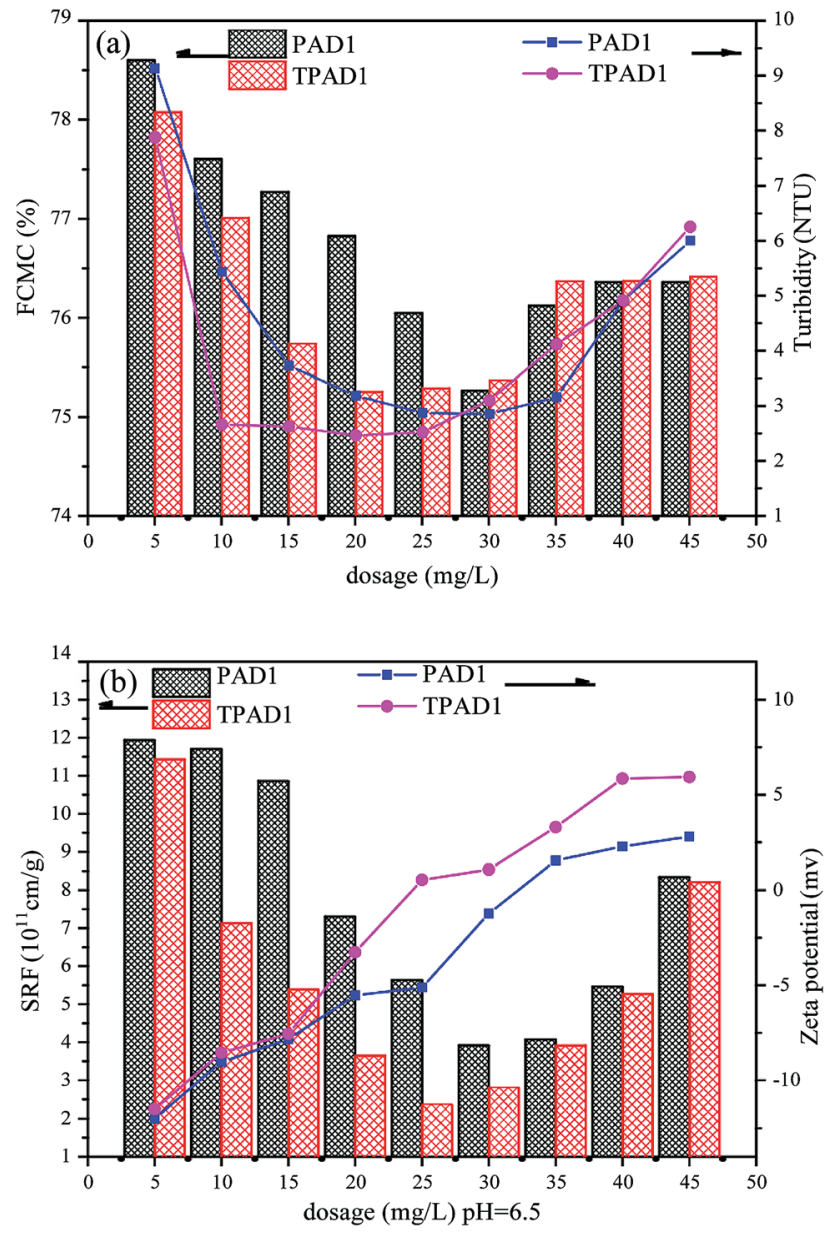

Fig. 10 The effect of dosage on (a) FCMC and turbidity, (b) SRF and zeta potential.

3.5.1 The effect of dosage. Fig. 10 shows the effect of dosage on FCMC, ST, SRF and zeta potential. The two flocculants presented similar trend as shown in Fig. 10. FCMC, ST and SRF all gradually decreased to minimum at the beginning, and then increased slowly. Consideration that almost identical intrinsic viscosity of the two flocculants diminished the difference in other dewatering mechanism such as the bridging and sweep effect. In this study, the charge neutralization effect of the two flocculants in dewatering process was investigated. Based on previous work, the change of zeta potential can well evaluate the dewatering mechanism especially the charge neutralization effect. ${ }^{49}$ The zeta potential was measured to explain above dewatering performance. In Fig. 10(b), the zeta potential shifted from the negative charge $(-14.06 \mathrm{mV}$ at dosage $\left.0 \mathrm{mg} \mathrm{L}^{-1}\right)$ to the positive charge $(2.8 \mathrm{mV}$ for PAD1 and $5.93 \mathrm{mV}$ for TPAD1 at dosage $45 \mathrm{mg} \mathrm{L}^{-1}$ ) with the increase of flocculants dosage. At insufficient flocculants dose, it was difficult to neutralize the excess negative charge of particles surface. With excess addition of the flocculants, sludge flocs would restabilize due to excess positive charge resulting in the electrostatic repulsion and steric hindrance among primary flocs., ${ }^{4,50}$ Therefore, low dosage or excess flocculants was not conducive to
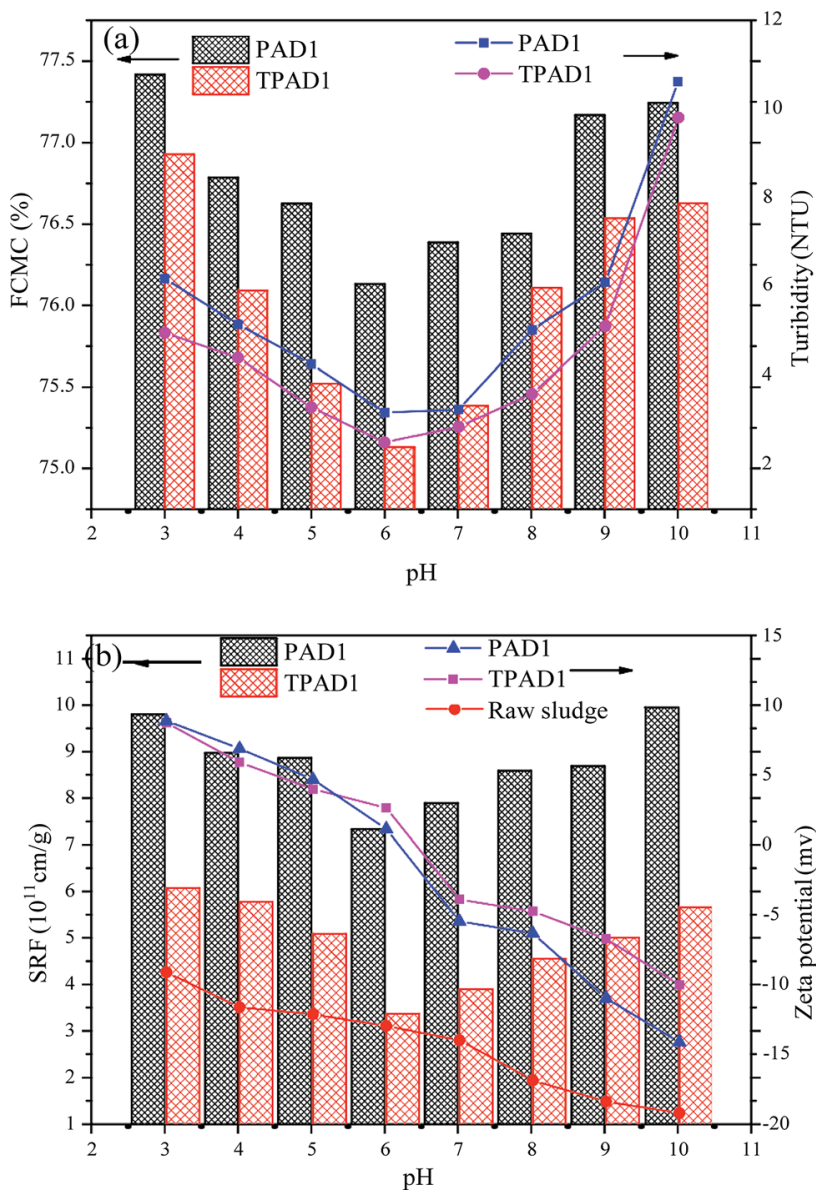

Fig. 11 The effect of pH on (a) FCMC and turbidity, (b) SRF and zeta potential.

sludge dewatering. In addition, the dewatering efficiency for PAD1 (75.26\% for FCMC, 2.84 NTU for ST, and $3.92 \times 10^{11} \mathrm{~cm}$ $\mathrm{g}^{-1}$ for SRF at dosage $\left.30 \mathrm{mg} \mathrm{L}^{-1}\right)$ was inferior to TPAD1 $(75.24 \%$ for FCMC, $2.45 \mathrm{NTU}$ for ST at dosage $20 \mathrm{mg} \mathrm{L}^{-1}$ and $2.35 \times$ $10^{11} \mathrm{~cm} \mathrm{~g}^{-1}$ for SRF at dosage $25 \mathrm{mg} \mathrm{L}^{-1}$ ). The result showed that the optimum dosage of TPAD1 was lower than PAD1. The porous structure and huge surface area of TPAD1 could effectively adsorb sludge particles and enhanced the bridging effect, thereby improving the dewatering effect.

3.5.2 The effect of $\mathbf{p H}$. As reported previously, $\mathrm{pH}$ is a critical factor for the sludge dewatering efficiency. ${ }^{51}$ Fig. 11 shows the effect of pH on FCMC, ST and SRF fixed flocculants dosage at $20 \mathrm{mg} \mathrm{\textrm {L } ^ { - 1 }}$. The FCMC, ST and SRF of the two flocculants gradually decreased when the $\mathrm{pH}$ changed from 3.0 to 6.0 and reached a minimum at $\mathrm{pH}=6.0$, whereas dewatering performance continually deteriorated with the $\mathrm{pH}$ increased from 6.0 to 10.0 . The result was consistent with Wang's report. ${ }^{49}$ Excessive acid or alkali environment went against the dewatering performance. The zeta potential was measured to further explain above phenomenon. In Fig. 11(b), when the $\mathrm{pH}$ changed from 3.0 to 10.0 , the zeta potential of raw sludge rapidly decreased from $-9.2 \mathrm{mV}$ to $-\mathbf{1 9 . 2 5} \mathrm{mV}$ and the supernatant zeta potential continually descended from $8.8 \mathrm{mV}$ to $-14.2 \mathrm{mV}$ (after 
treatment with PAD1), from $8.6 \mathrm{mV}$ to $-10.1 \mathrm{mV}$ (after treatment with TPAD1). Under strong acid $(\mathrm{pH}=3.0,4.0)$, excess positive charge $\left(\mathrm{H}^{+}\right)$enclosed in colloidal particles, which increased the electrostatic repulsion between colloidal particles and cationic polymer, resulting in too high zeta potential (above $5.8 \mathrm{mV}$ ) and the weak charge neutralization effect. Under strong alkali $(\mathrm{pH}=9.0,10.0)$, excess negative charge $\left(\mathrm{OH}^{-}\right)$consumed some of cationic flocculants, then caused too low zeta potential (below $-6.8 \mathrm{mV}$ ) and weakened the charge neutralization effect. When $\mathrm{pH}$ was near 6.0 , the zeta potential was close to the isoelectric point. This indicated that the charge neutralization effectively occurred in dewatering process and then the surface charge of colloidal particles went down to zero. Hence, the optimum $\mathrm{pH}$ value is 6.0-7.0.

3.5.3 Sludge floc properties. The size of sludge floc is closely related to the dewatering performance. Generally, small sludge floc is more likely to block the filter holes resulting in the increase of filtration resistance, thus increasing the subsequent processing cost. On the contrary, larger floc is easier to settle and dewatering, which can directly reflect the dewatering efficiency of flocculants. ${ }^{52}$ In order to further investigate the dewatering behavior, the size distribution and morphology of sludge flocs are shown in Fig. S2. $\dagger$ The flocculants dose was $30 \mathrm{mg} \mathrm{L}{ }^{-1}$ of PAD1, $20 \mathrm{mg} \mathrm{L^{-1 }}$ of TPAD1 and the $\mathrm{pH}$ was adjusted to 6.0. As shown in Fig. S2, $\dagger$ the floc size was $d_{50}=$ $742.464 \mu \mathrm{m}$ of PAD1 and $d_{50}=1004.051 \mu \mathrm{m}$ of TPAD1. The result indicated that TPAD1 with excellent dewatering efficiency presented larger floc size, which was in accordance with the above experimental phenomenon. In addition, Fig. S2 $\uparrow$ shows the floc morphology after treatment with PAD1 and TPAD1 conditioning. Obviously, the formed sludge flocs of TPAD1 were dense and strong granules, which had fast sedimentation velocity and high resistance for the elevated hydraulic shear force.$^{53}$ However, the morphology of sludge flocs after treatment with PAD1 was loose and fragile granules. The results further proved that the synthesized flocculants by the photocatalytic surface initiation method showed superior dewatering performance compared with PAD1 and CPAM.

\section{Conclusion}

In this study, cationic polymeric flocculants (TPADs) were synthesized by photocatalytic surface initiation using nano$\mathrm{TiO}_{2}$ as an initiator. The successful copolymerization was confirmed by characterization analysis such as FTIR, ${ }^{1} \mathrm{H}$ NMR and TG/DSC. Results indicated that the TPADs had a distinct cationic sequence distribution, porous surface morphology and prodigious surface area. Two types of initiation mechanism in polymerization were outlined and the study of the flocculants' properties confirmed that TPADs had extra-high intrinsic viscosity. The flocculation test showed that TPADs exhibited excellent low turbidity and $\mathrm{UV}_{254}$ removal efficiency in a wide range of $\mathrm{pH}(\mathrm{pH} 3.0-9.0)$. When the dosage of TPAD2 was $6 \mathrm{mg} \mathrm{L}^{-1}$, the best flocculation performance (93.79\% of TRE and 0.08 of $\mathrm{UV}_{254}$ ) was acquired. A dewatering test demonstrated that the optimum dewatering performance by TPAD1 was 2.45 NTU of ST and $75.24 \%$ of FCMC at a dosage of $20 \mathrm{mg} \mathrm{L}^{-1}$, and
$2.35 \times 10^{11} \mathrm{~cm} \mathrm{~g}^{-1}$ of SRF at a dosage of $25 \mathrm{mg} \mathrm{L}^{-1}$. Besides, the sludge flocs of TPAD1 showed a compact morphology and larger size, which further confirmed that TPAD1 had a superior dewatering ability to PAD1. The excellent flocculation/dewatering performance should be attributed to the combination of the photocatalytic surface-initiated method with the synthesis of cationic polymeric flocculant.

\section{Conflicts of interest}

There are no conflicts to declare.

\section{Abbreviations}

$\begin{array}{ll}\text { AM } & \text { Acrylamide } \\ \text { DAC } & \text { Acryloyloxyethyl trimethylammonium chloride } \\ \text { CPAMs } & \text { Cationic polyacrylamides } \\ \text { TPADs } & \text { Synthesized cationic polymeric flocculants by } \\ \text { (TPAD1-6) } & \begin{array}{l}\text { photocatalytic surface-initiated polymerization } \\ \text { using nano- } \mathrm{TiO}_{2} \text { as initiator }\end{array} \\ & \text { Synthesized cationic polymeric flocculants by } \\ \text { PADs } & \text { UV-initiated method using VA-044 as photo } \\ \text { (PAD1-3) } & \text { initiator } \\ & \text { Commercial cationic polyacrylamide } \\ \text { CPAM } & \text { Fourier transform infrared spectroscopy } \\ \text { FTIR } & \text { Proton nuclear magnetic resonance }\end{array}$

spectrum

TG/DSC Thermal analysis

SEM Scanning electron microscopy

VA-044 2,2'-Azobis [2-(2-imidazolin-2-yl) propane]

UV Ultraviolet

TRE Turbidity removal efficiency

ST Supernatant turbidity

FCMC Filter cake moisture content

SRF Specific resistance to filtration

$\mathrm{UV}_{254} \quad$ Absorbance in $254 \mathrm{~nm}$ by ultraviolet

spectrometry

\section{Acknowledgements}

This work was supported by the National Natural Science Foundation of China (Project No. 21477010), 111 Project (Project No. B13041) and Graduate Research and Innovation Foundation of Chongqing, China (Project No. CYB18041).

\section{References}

1 Z. Z. Liu, H. Wei, A. M. Li and H. Yang, Evaluation of structural effects on the flocculation performance of a cograft starch-based flocculant., Water Res., 2017, 118, 160166.

2 C. L. Zhao, H. L. Zheng, L. Feng, Y. L. Wang, Y. Z. Liu, B. Z. Liu and B. Djibrine, Improvement of sludge dewaterability by ultrasound-initiated cationic 
polyacrylamide with microblock structure: The role of surface-active monomers, Materials, 2017, 10, 282.

3 H. L. Zheng, L. Feng, B. Y. Gao, Y. H. Zhou and S. S. Zhang, Effect of the cationic block structure on the characteristics of sludge flocs formed by charge neutralization and patching, Materials, 2017, 487.

4 Y. Liao, H. L. Zheng, Q. Li, Y. J. Sun, D. Li and W. W. Xue, UVinitiated polymerization of hydrophobically associating cationic polyacrylamide modified by a surface-sctive monomer: a comparative study of synthesis, characterization, and sludge dewatering performance, Ind. Eng. Chem. Res., 2014, 53, 11193-11203.

5 X. Huang, B. Y. Gao, H. Y. Rong, Q. Y. Yue, Y. Y. Zhang and P. Y. Teng, Effect of using polydimethyldiallylammonium chloride as coagulation aid on polytitanium salt coagulation performance, floc properties and sludge reuse, Sep. Purif. Technol., 2015, 143, 64-71.

6 R. H. Li, B. Y. Gao, K. Y. Guo, Q. Y. Yue, H. L. Zheng and Y. Wang, Effects of papermaking sludge-based polymer on coagulation behavior in the disperse and reactive dyes wastewater treatment, Bioresour. Technol., 2017, 240, 59-67.

7 D. H. Yoon, J. W. Jang and I. W. Cheong, Synthesis of cationic polyacrylamide/silica nanocomposites from inverse emulsion polymerization and their flocculation property for papermaking, Colloids Surf., A, 2012, 411, 18-23.

8 L. Lévesque, C. A. Mizzen, D. R. Mclachlan and P. E. Fraser, Ligand specific effects on aluminum incorporation and toxicity in neurons and astrocytes., Brain Res., 2000, 877, 191-202.

9 L. H. Lu, Z. D. Pan, H. Nan and W. Q. Peng, A novel acrylamide-free flocculant and its application for sludge dewatering, Water Res., 2014, 57, 304-312.

10 R. Yang, H. J. Li, M. Huang, H. Yang and A. M. Li, A review on chitosan-based flocculants and their applications in water treatment, Water Res., 2016, 95, 59-89.

11 Y. Bao, J. Z. Ma and N. Li, Synthesis and swelling behaviors of sodium carboxymethyl cellulose-g-poly(AA-co-AM-coAMPS)/MMT superabsorbent hydrogel, Carbohydr. Polym., 2011, 84, 76-82.

12 C. J. Zou, T. Gu, P. F. Xiao, T. T. Ge, M. Wang and K. Wang, Experimental study of cucurbit[7]uril derivatives modified acrylamide polymer for enhanced oil recovery, Ind. Eng. Chem. Res., 2014, 53, 7570-7578.

13 X. Jiang, Y. Y. Yin, C. Y. Wang and X. Z. Tian, Decolorization of anionic dye solutions using the hydrophobically modified polyelectrolytes containing beta-cyclodextrin moieties, Chem. Eng. J., 2014, 253, 183-189.

14 C. L. Zhao, H. L. Zheng, B. Y. Gao, Y. Z. Liu, J. Zhai, S. X. Zhang and B. C. Xu, Ultrasound-initiated synthesis of cationic polyacrylamide for oily wastewater treatment: Enhanced interaction between the flocculant and contaminants, Ultrason. Sonochem., 2018, 42, 31-41.

15 Y. J. Sun, C. Y. Zhu, W. Q. Sun, Y. H. Xu, X. F. Xiao, H. L. Zheng, H. F. Wu and C. Y. Liu, Plasma-initiated polymerization of chitosan-based CS-g-P(AM-DMDAAC) flocculant for the enhanced flocculation of low-algalturbidity water, Carbohydr. Polym., 2017, 164, 222-232.
16 Y. H. Zhou, H. L. Zheng, B. Y. Gao, Y. P. Gu, X. Li, B. Z. Liu and A. M. Jiménez, Waste activated sludge (WAS) dewatering properties of an original hydrophobically modified polyacrylamide containing a cationic microblock structure, RSC Adv., 2017, 7, 28733-28745.

17 X. Li, H. L. Zheng, B. Y. Gao, Y. J. Sun, B. Z. Liu and C. L. Zhao, UV-initiated template copolymerization of AM and MAPTAC: Microblock structure, copolymerization mechanism, and flocculation performance, Chemosphere, 2017, 167, 71-81.

18 X. Wang, Q. P. Lu, X. F. Wang, J. B. Joo, M. Dahl, B. Liu, C. B. Gao and Y. D. Yin, Photocatalytic surface-initiated polymerization on $\mathrm{TiO}_{2}$ toward well defined composite nanostructures, ACS Appl. Mater. Interfaces, 2016, 8, 538-546.

19 X. Y. Ni, J. Ye and C. Dong, Kinetics studies of methyl methacrylate photopolymerization initiated by titanium dioxide semiconductor nanoparticles, J. Photochem. Photobiol., A, 2006, 181, 19-27.

20 D. Zhang, J. H. Yang, S. Bao, Q. S. Wu and Q. G. Wang, Semiconductor nanoparticle-based hydrogels prepared via self-initiated polymerization under sunlight, even visible light, Sci. Rep., 2013, 3, 1399.

21 H. L. Zheng, J. Y. Ma, C. J. Zhu, Z. Zhang, L. W. Liu, Y. J. Sun and X. M. Tang, Synthesis of anion polyacrylamide under UV initiation and its application in removing dioctyl phthalate from water through flocculation process, Sep. Purif. Technol., 2014, 123, 35-44.

22 C. Becker-Willinger, S. Schmitz-Stoewe, D. Bentz and M. Veith, in Kinetic investigations on $\mathrm{TiO}_{2}$ nanoparticles as photo initiators for UV-polymerization in acrylic matrix, ed. M. A. Maher, J. C. Chiao and P. J. Resnick, San Francisco, 2010, p. 7590.

23 C. N. Liao, Q. Wu, T. Su, D. Zhang, Q. S. Wu and Q. G. Wang, Nanocomposite gels via in situ photoinitiation and disassembly of $\mathrm{TiO}_{2}$-clay composites with polymers applied as UV protective films, ACS Appl. Mater. Interfaces, 2014, 6, 1356-1360.

24 H. Kong, J. Song and J. Jang, Photocatalytic antibacterial capabilities of $\mathrm{TiO}_{2}$-biocidal polymer nanocomposites synthesized by a surface-initiated photopolymerization, Environ. Sci. Technol., 2010, 44, 5672-5676.

25 X. X. Wang, X. M. Song, M. Lin, H. T. Wang, Y. L. Zhao, W. Zhong and Q. G. Du, Surface initiated graft polymerization from carbon-doped $\mathrm{TiO}_{2}$ nanoparticles under sunlight illumination, Polymer, 2007, 48, 5834-5838.

26 D. Ding, K. Liu, S. He, C. Gao and Y. Yin, Ligand-exchange assisted formation of $\mathrm{Au} / \mathrm{TiO}_{2}$ schottky contact for visiblelight photocatalysis, Nano Lett., 2014, 14, 6731.

27 H. L. Zheng, Y. J. Sun, J. S. Guo, F. T. Li, W. Fan, Y. Liao and Q. Q. Guan, Characterization and evaluation of dewatering properties of PADB, a highly efficient cationic flocculant, Ind. Eng. Chem. Res., 2014, 53, 2572-2582.

28 Y. X. Zhao, B. Y. Gao, G. Z. Zhang and Q. B. Qi, Coagulation and sludge recovery using titanium tetrachloride as coagulant for real water treatment: A comparison against traditional aluminum and iron salts, Sep. Purif. Technol., 2014, 130, 19-27. 
29 X. Li, H. L. Zheng, B. Y. Gao, C. Zhao and Y. J. Sun, UVinitiated polymerization of acid- and alkali-resistant cationic flocculant P(AM-MAPTAC): Synthesis, characterization, and application in sludge dewatering, Sep. Purif. Technol., 2017, 187, 244-254.

30 H. L. Zheng, Y. J. Sun, C. J. Zhu, J. S. Guo, C. Zhao, Y. Liao and Q. Q. Guan, UV-initiated polymerization of hydrophobically associating cationic flocculants: Synthesis, characterization, and dewatering properties, Chem. Eng. J., 2013, 234, 318-326.

31 Q. Q. Guan, H. L. Zheng, J. Zhai, B. Z. Liu, Y. J. Sun, Y. L. Wang, Z. N. Xu and C. Zhao, Preparation, characterization, and flocculation performance of $\mathrm{P}$ (acrylamide-co-diallyldimethylammonium chloride) by UV-initiated template polymerization, J. Appl. Polym. Sci., 2015, 132, 41747.

32 B. Z. Liu, H. L. Zheng, X. R. Deng, B. C. Xu, Y. J. Sun, Y. Z. Liu and J. J. Liang, Formation of cationic hydrophobic microblocks in $\mathrm{P}(\mathrm{AM}-\mathrm{DMC})$ by template assembly: characterization and application in sludge dewatering, $R S C$ Adv., 2017, 7, 6114-6122.

33 P. Pal, J. P. Pandey and G. Sen, Synthesis of polyacrylamide grafted polyvinyl pyrollidone (PVP-g-PAM) and study of its application in algal biomass harvesting, Ecol. Eng., 2017, 100, 19-27.

34 W. Chen, H. L. Zheng, Q. Q. Guan, H. K. Teng, C. L. Zhao and C. Zhao, Fabricating a Flocculant with Controllable Cationic Microblock Structure: Characterization and Sludge Conditioning Behavior Evaluation, Ind. Eng. Chem. Res., 2016, 55, 2892-2902.

35 B. Kordoghli, R. Khiari, M. F. Mhenni, F. Sakli and M. N. Belgacem, Sulfonation of polyester fabrics by gaseous sulfur oxide activated by UV irradiation, Appl. Surf. Sci., 2012, 258, 9737-9741.

36 Z. Xiao, J. B. Zhu, W. W. Cai, M. L. Xiao, L. Liang, C. P. Liu and $\mathrm{X}$. Wei, Pt-Pb hollow sphere networks: self-sacrificetemplating method and enhanced activity for formic acid electrooxidation, RSC Adv., 2013, 3, 1763-1767.

37 P. Wu, N. Du, H. Zhang, C. Zhai and D. Yang, SelfTemplating Synthesis of $\mathrm{SnO}_{2}$-Carbon Hybrid Hollow Spheres for Superior Reversible Lithium Ion Storage, ACS Appl. Mater. Interfaces, 2011, 3, 1946-1952.

38 C. Liu, L. Wang, Y. Tang, S. Luo, Y. Liu, S. Zhang, Y. Zeng and Y. Xu, Vertical single or few-layer $\mathrm{MoS}_{2}$ nanosheets rooting into $\mathrm{TiO}_{2}$ nanofibers for highly efficient photocatalytic hydrogen evolution, Appl. Catal., B, 2015, 164, 1-9.

39 C. Dong and X. Y. Ni, The photopolymerization and characterization of methyl methacrylate initiated by nanosized titanium dioxide, J. Macromol. Sci., Part A: Pure Appl.Chem., 2004, A41, 547-563.

40 D. Trimnell, G. F. Fanta and J. H. Salch, Graft polymerization of methyl acrylate onto granular starch: Comparison of the $\mathrm{Fe}^{+2} / \mathrm{H}_{2} \mathrm{O}_{2}$ and ceric initiating systems, J. Appl. Polym. Sci., 2015, 60, 285-292.
41 W. Brostow, S. Pal and R. P. Singh, A model of flocculation, Mater. Lett., 2007, 61, 4381-4384.

42 P. Pal, J. P. Pandey and G. Sen, Modified PVP based hydrogel: Synthesis, characterization and application in selective abstraction of metal ions from water, Mater. Chem. Phys., 2017, 194, 261-273.

43 Z. Yang, H. Yang, Z. W. Jiang, T. Cai, H. J. Li, H. B. Li, A. M. Li and R. S. Cheng, Flocculation of both anionic and cationic dyes in aqueous solutions by the amphoteric grafting flocculant carboxymethyl chitosan-graft-polyacrylamide, $J$. Hazard. Mater., 2013, 254-255, 36.

44 X. Li, H. L. Zheng, B. Y. Gao, Y. J. Sun, X. M. Tang and B. C. Xu, Optimized preparation of micro-block CPAM by response surface methodology and evaluation of dewatering performance, RSC Adv., 2017, 7, 208-217.

45 X. Lu, Y. H. Xu, W. Q. Sun, Y. J. Sun and H. L. Zheng, UVinitiated synthesis of a novel chitosan-based flocculant with high flocculation efficiency for algal removal, Sci. Total Environ., 2017, 609, 410-418.

46 J. Y. Ma, J. Shi, H. C. Ding, G. C. Zhu, K. Fu and X. Fu, Synthesis of cationic polyacrylamide by low-pressure UV initiation for turbidity water flocculation, Chem. Eng. J., 2017, 312, 20-29.

47 Z. A. Zhang, H. L. Zheng, F. Huang, X. Li, S. Y. He and C. Zhao, Template Polymerization of a Novel Cationic Polyacrylamide: Sequence Distribution, Characterization, and Flocculation Performance, Ind. Eng. Chem. Res., 2016, 55, 9819-9828.

48 J. C. Wei, B. Y. Gao, Q. Y. Yue and Y. Wang, Strength and regrowth properties of polyferric-polymer dual-coagulant flocs in surface water treatment, J. Hazard. Mater., 2010, 175, 949-954.

49 W. Y. Wang, Q. Y. Yue, R. H. Li, W. Song, B. Y. Gao and $\mathrm{X}$. Shen, Investigating coagulation behavior of chitosan with different $\mathrm{Al}$ species dual-coagulants in dye wastewater treatment, J. Taiwan Inst. Chem. Eng., 2017, 78, 423-430.

50 L. Feng, H. L. Zheng, Y. L. Wang, S. X. Zhang and B. C. Xu, Ultrasonic-template technology inducing and regulating cationic microblocks in CPAM: characterization, mechanism and sludge flocculation performance, $R S C$ Adv., 2017, 7, 23444-23456.

51 H. L. Peng, S. X. Zhong, J. X. Xiang, Q. T. Lin, C. Yao, J. H. Dong, G. C. Yin, K. Yao, S. Y. Zeng and J. Zhong, Characterization and secondary sludge dewatering performance of a novel combined aluminum-ferrousstarch flocculant (CAFS), Chem. Eng. Sci., 2017, 173, 335-345.

52 Y. Q. Zhao, Settling behaviour of polymer flocculated watertreatment sludge II: effects of floc structure and floc packing, Sep. Purif. Technol., 2004, 35, 175-183.

53 J. Y. Ma, J. Shi, L. Ding, H. W. Zhang, S. Zhou, Q. J. Wang, $\mathrm{X} . \mathrm{Fu}, \mathrm{L} . \mathrm{Y}$. Jiang and $\mathrm{K}$. Fu, Removal of emulsified oil from water using hydrophobic modified cationic polyacrylamide flocculants synthesized from low-pressure UV initiation, Sep. Purif. Technol., 2018, 197, 407-417. 